\title{
Real-Time Obstacle Avoidance Using Harmonic Potential Functions *
}

\author{
Jin-Oh $\mathrm{Kim}^{\dagger} \quad$ and $\quad$ Pradeep K. Khosla ${ }^{\ddagger}$
}

'This research was funded in pan by NASA under grant NAG-1-1075, DOE under grant DE-F902-89ER 14042, the Department of Electrical and Computer Engineering, and the Robotics Institute, Carnegie-Mellon University.

IJ. O. Kim is with the Robotics Ph.D. Program. the Robotics Institute, Carnegie-Mellon University, Pittsburgh, PA, 15213

'P. K. Khosla is with the Department of Electrical and Computer Engineering and the Robotics Institute, Carnegie. Mellon University, Pittsburgh,PA, 15213. 


\begin{abstract}
This paper presents a new formulation of the artificial potential approach to the obstacle avoidance problem for a mobile robot or a manipulator in a known environment. Previous formulations of artificial potentials, for obstacle avoidance, have exhibited local minima in a cluttered environment. To build an artificial potential field, we use harmonic functions which completely eliminate local minima even for a cluttered environment. We use the panel method to represent arbitrarily shaped obstacles and to derive the potential over the whole space. Based on this potential function, we propose an elegant control strategy for the real-time control of a robot. We test the harmonic potential, the panel method and the control strategy with a bar-shaped mobile robot and a 3 dof planar redundant manipulator.
\end{abstract}




\section{Introduction}

The issue of obstacle avoidance in aknown environment has been addressed by many researchers [ 1 , $2,5,6,9,10,11,12,19]$. The proposed strategies are applicable to both mobile robots and manipulators and can be divided into two broad classes: global and local. The global techniques[ 14, 3] are guaranteed to give a free path if such a path exists. However, their computation time increases exponentially as the degrees of freedom (dof) of the robot increase [2]. This computational complexity limits their use for rea-time obstacle avoidance to only very simple cases. Local strategies are typically based on the artificial potential function approach and can be implemented either in task space $[10,11,12,13,211$ or in configuration space $[17,19,223$.

In the artificial potential approach, the obstacles to be avoided are represented by a repulsive artificial potential and the goal is represented by an attractive potential, so that a robot reaches the goal without colliding with obstacles. This approach is computationally much less expensive than the global approach and is therefore suited for real-time implementation. The artificial potential approach, however, has been limited due to the existence of local minima and its inability to deal with arbitrarily shaped obstacles. Recently, the superquadric potential function has been proposed to model obstacles with arbitrary shapes[12,211. In [19], it has been shown that the local minima can be removed with limited success in configuration space but at the cost of increased computational complexity. The advantage of fast computation of the potential function approach has been augmented with search methods to avoid local minima in complex environments [2], but at the cost of increased precomputation time.

In this paper, we introduce a novel artificial potential function, based on harmonic functions, that overcomes the limitations of previous formulations. The most important property of harmonic functions is that they are free from local minima [8]. This property was used in [1] with limited success for simple environment and in [6] with numerical approach for planning. Our approach generalizes the previous approach and is composed of two steps. In the first step, we build an artificial potential and in the second step we develop a control strategy for navigating in the potential field. To build an artificial potential, we use harmonic functions, which do not have any local extrema in a space free from singularities. To derive the potential field for obstacle avoidance, we use the panel method with harmonic functions. The panel method has been used to solve the potential flow problem of a fluid around an arbitrarily shaped body in both $\mathbf{2}$ and 3 dimensional task spaces [4, 7]. Even though the potential field obtained using harmonic functions and panel method is perfect for a point mobile robot, it does not guarantee success for non-point robots. To overcome this, we develop a control strategy for non-point robots. In this paper, we present simulation results demonstrating our approach using two examples: a bar-shaped mobile robot and a $\mathbf{3}$ degree-of-freedom (dof) planar redundant manipulator.

This paper is organized as follows; In Section 2 we introduce properties of harmonic function, and compare harmonic function and non-harmonic function. In Section 3, we introduce panel method to build potential field over the whole task space. In Section 4, We discuss differences between real potential and potential used for obstacle avoidance. In Section 5, we derive control strategy for non-point robots and simulation results are shown. In Section 6, we discuss limitation of our approach and this paper is concluded in Section 7. 


\section{Potential Theory and Harmonic Function}

In this section, the theory of incompressible potential flow is introduced $[4,7,16]$. In the absence of viscous effects and rotational force, the originally irrotational flow far upstream will remain so in the region around a body inside the flow field. Letting $V$ denote the velocity field in this region, the irrotationality condition states that the vorticity vanishes. That is,

$$
\text { Vorticity }=\text { curl } V=\nabla \times V=0 \text {. }
$$

This equation implies that the velocity field can be written as

$$
V=-\nabla \phi,
$$

where $\phi$ is a scalar velocity potential.

Furthermore, when the fluid is incompressible, the velocity field must satisfy the continuity equation;

$$
\nabla \cdot V=0
$$

Upon substitution of (2) into (3), we get

$$
\nabla^{2} \phi=0
$$

where $\nabla^{2} \equiv \mathrm{V} \cdot \mathrm{V}$ is the Laplacian operator.

Equation (4) is called Laplace equation or potential equation, and its solutions are called harmonic functions or potential functions. In the real world, many physical problems are described by the Laplace equation. An example is the incompressible potential flow as mentioned above. A steady state temperature dismbution also follows this Laplace equation. For this case, the temperature becomes the potential function. A steady state electric charge distribution is another example.

\subsection{Properties of Harmonic Function}

The first important property of a harmonic function is the principle of superposition, which follows from the linearity of the Laplace equation. That is, if $\phi_{1}$ and $\phi_{2}$ are harmonic, then any linear combination of $\phi_{1}$ and $\phi_{2}$ is also harmonic and a solution of the Laplace equation.

Other important properties of harmonic functions related to local minimum are as follows: 


\section{Property 1 : Mean-Value Property}

For a 2 dimensional potential function $\phi(x, y)$ which is harmonic inside a circle centered at $\left(x_{0}, y_{0}\right)$, there exists the mean-value property of $\phi$;

$$
\phi\left(x_{0}, y_{0}\right)=\frac{1}{2 \pi} \oint_{0}^{2 \pi} \phi\left(x_{0}+r \cos \theta, y_{0}+r \sin \theta\right) d \theta
$$

This property is independent of the radius $r$ of the circle only if the potential function $\circ$ is harmonic inside the circle.

The proof of this property can be found in [8]. A similar result holds for an arbitrary number of dimensions. For example, in three dimensions, the potential at $\left(x_{0}, y_{0}, z_{0}\right)$ can be obtained by integration over the whole surface $S$ of a sphere:

$$
\phi\left(x_{0}, y_{0}, z_{0}\right)=\frac{1}{4 \pi} \iint \phi(S) d S .
$$

The converse of Property 1 is also true. That is, if $\phi(x, y)$ is continuous and has the mean-value property for every circle in a domain, then $\phi$ is harmonic. The above mean-value property is an intrinsic property of a harmonic function. This property can be used to prove the maximum and minimum principle of harmonic funcrions.

\section{Property 2 : The Maximum Principle}

The maximum of a nonconstant harmonicfunction occurs on the boundary.

Proof: Suppose that $\phi$ is a nonconstant harmonicfunction and that the maximum $M$ of $\phi$ occurs at a non-boundary point $P$. By imagining a circle centered at $P$ inside of the boundary, we can easily prove that the above assumption contradicts the mean-value property in Property 1, because the point $P$ must have mean value of the integration along the contour of the circle.

$1 / 1$

\section{Property 3 : The Minimum Principle}

The minimum of a nonconstant harmonicfunction also occurs on the boundary,

Proof: Apply the proof of Property 2 to $-\phi$.

The above properties of a harmonic function are very useful in building an artificial potential field for the obstacle avoidance problem because the harmonic function completely eliminates local minima. These properties were used for obstacle avoidance in $[1,6]$. The use of complex variable and conformal mapping in [1] may be useful for teel-time control in a simple environment. However, the complex variable approach cannot be extended for $\mathbf{3}$ dimensional space and the conformal mapping method fails for multi obstacles and an arbitrarily shaped obstacle. The numerical approach in [6] is staightforward and appropriate for a global path planning problem and for a bounded environment. This gives artificial potential at finite number of points in a free space. On the other hand, our analytical approach in this paper is appropriate for both $\mathbf{2}$ and 3 dimensional spaces and for both.real-time control and global path planning because it provides a closed form artificial potential over the whole space. Furthermore, our approach can be applied for both bounded and unbounded environments. 


\subsection{Examples of Harmonic Function}

In this section, we introduce examples of harmonic functions which will be used later to build an artificial potential. First, harmonic functions with spherical symmetry are introduced from [8]. These functions depend only on $\boldsymbol{r}$ (distancefrom the origin). The general $\mathrm{n}$ dimensional expression of the Laplace equation (4) in a polar coordinate can be written as:

$$
\nabla^{2} \phi=\phi_{r r}+\frac{n-1}{r} \phi_{r}+\text { angular terms, }
$$

where $\phi_{r r}$ is the second partial derivative of $\phi$ with respect to $r$ and $\phi_{r}$ is the first partial derivative with respect to $r$. Since $\phi=\phi(r)$, the angular terms in (5)become zero. Then, the Laplace equation becomes

$$
\begin{aligned}
& \phi_{r r}+\frac{n-1}{r} \phi_{r}=0 \\
& \text { or } \\
& \frac{\phi_{r r}}{\phi_{r}}+\frac{n-1}{r}=0 .
\end{aligned}
$$

Integrating (7) once, we obtain

$$
\phi_{r}=\frac{c_{1}}{r^{n-1}}
$$

For $n=2$, the solution of Equation (8) is

$$
\phi=c_{1} \log r+c_{2}
$$

If $\mathrm{n}>\mathbf{2}$, then the solution is

$$
\phi=\frac{c_{3}}{r^{n-2}}+c_{4} .
$$

In Equations (8), (9) and (10), $c_{i}$ 's are constant. From Equations (9)and (10), we observe that every harmonic function with spherical symmetry has its singularity at the origin and is not harmonic at this singular point. Since the origin can be placed anywhere (the Laplace equation is invariant under translation), we can always choose the origin outside of the free space for a manipulator and a mobile robot. That is, by locating origins of the harmonic functions on the surface of obstacles or inside obstacles, we can build an artificial potential field with no local minimum and one global minimum in free space.

In hydrodynamics, the above harmonic function with spherical symmetry is called source or sink depending on the sign of $c_{1}$ in (9) and $c_{3}$ in (10). A sink is like a drain in a bathtub and a source is like a faucet. In $\mathbf{2}$ dimensions, a source/sink at the origin can be represented by:

$$
\phi=\frac{\lambda}{2 \pi} \log r .
$$


The magnitude of $\lambda$ is called the strength of the source $(\lambda<0)$ or the sink $(\lambda>0)$. Since both source and sink are singular at origin, they are called singularities. Note that the general $n$ dimensional harmonic function in (10) enables the extension of our approach to $n$ dimensional configuration spaces.

Another harmonic function useful for building artificial potentials is uniform flow, whose potential varies linearly along the direction of flow. In $\mathbf{2}$ dimensions, when the fluid flows in a direction which makes an angle $\alpha$ with the x-axis, the potential function for this uniform flow is

$$
\phi=-U(x \cos \alpha+y \sin \alpha) .
$$

The magnitude of coefficient $U$ is called the strength of uniform flow.

In this paper, we use source/sink singularitiesin (11)to derive the repulsive force (high potential) of obstacles and the attractive force (low potential) of a goal position, and the uniform flow in (12) to derive a more effective artificial potential field from a starting position to a goal position. This uniform flow provides a linearly decreasing potential in the direction from a starting position to a goal position for the unbounded environment.

\subsection{Harmonic Function vs. Non-Harmonic Function}

Harmonic and non-harmonic functions for the $\mathbf{2}$ dimensional case are compared in this subsection. One harmonic function with spherical symmetry for $\mathbf{2}$ dimensions is $-\log r$. An example of a non-harmonic function for $\mathbf{2}$ dimensions is $1 / \boldsymbol{r}$. Note that $1 / r$ is harmonic for $\mathbf{3}$ dimensions but not harmonic for $\mathbf{2}$ dimensions as can be seen $\operatorname{fram}(10)$. The function $1 / r$ has been used for the 2 dimensional case in $[2,12]$.

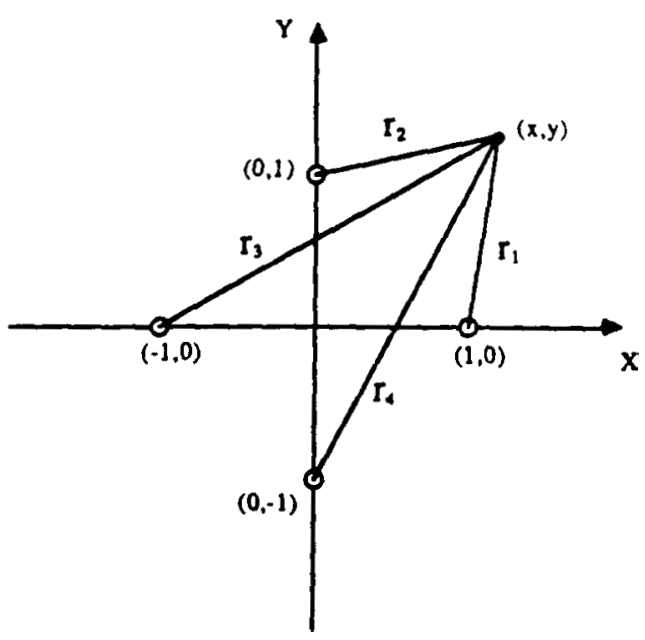

Figure 1: Locations of Singularities

Suppose that there are 4 point obstacles at $(1,0),(-1,0),(0,1)$ and $(0,-1)$ as shown in Figure 1 . The distance $r_{i}$ is measured from point obstacle $i$ to $(x, y)$. With a harmonic function, the artificial 
potential field can be expressed as

$$
\phi_{1}(x, y)=-\log r_{1}-\log r_{2}-\log r_{3}-\log r_{4}
$$

And the artificial potential field with a non-harmonic function $(1 / r)$ is

$$
\phi_{2}(x, y)=\frac{1}{r_{1}}+\frac{1}{r_{2}}+\frac{1}{r_{3}}+\frac{1}{r_{4}}
$$

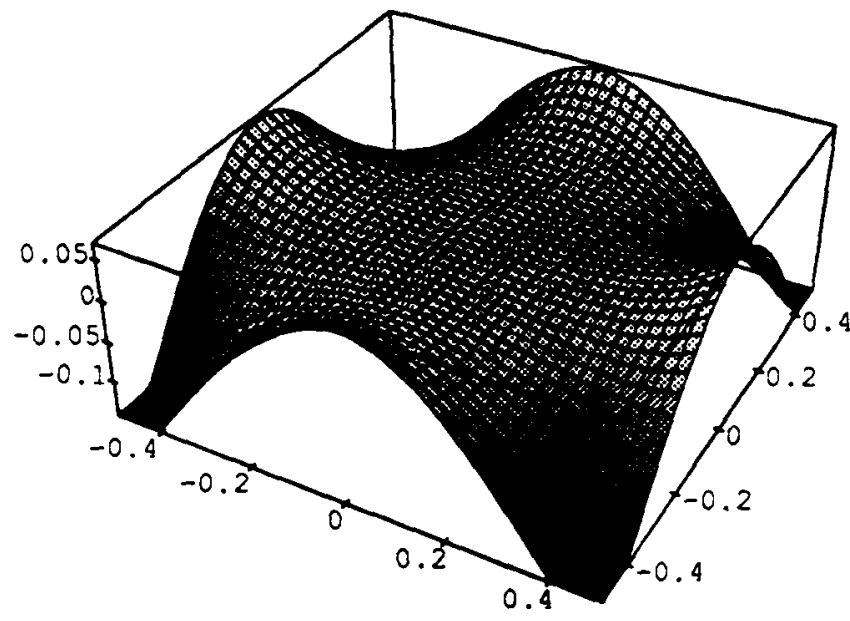

(a)

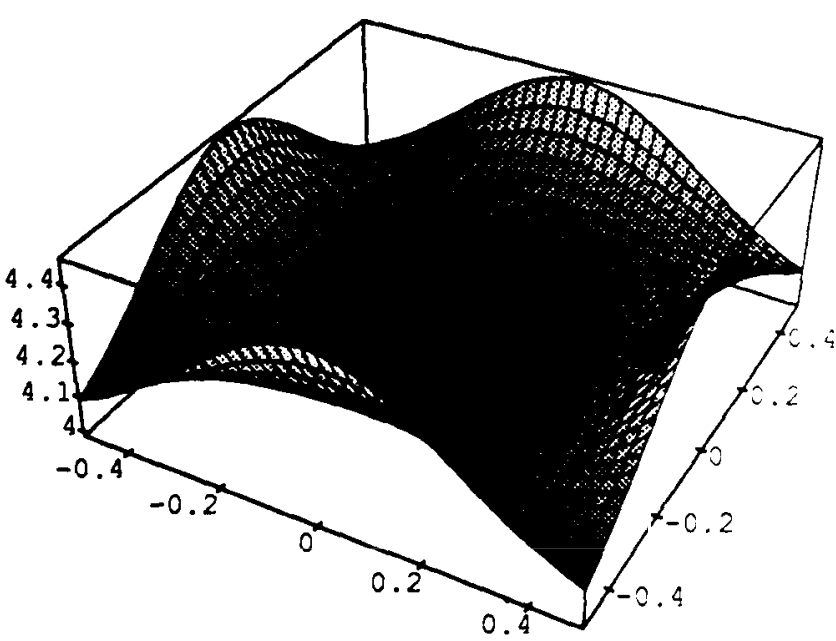

(b)

Figure 2: (a) Harmonic Function (b) Non-Harmonic Function

Figures 2(a) and 2(b) show the artificial potential field of $\phi_{1}$ (harmonic function) and $\phi_{2}$ (nonharmonic function), respectively. There exists a local minimum at $(0,0)$ for the non-harmonic function but there does not exist a local minimum for the harmonic function. In Figure 2(a), the origin $(0,0)$ is called a stagnation point in hydrodynamics, where the velocity of fluid particle becomes zero instantaneously. But this stagnation point is an unstable saddle point, not a local minimum.

To extend the observation from this comparison, we can conclude that the non-harmonic function is vulnerable to local minimum. Anotherexample is the function $1 / \boldsymbol{r}^{2}$ in [11], which is not harmonic for $\mathbf{2}$ dimensions, but harmonic for $\mathbf{4}$ dimensions. Here we assert that we have to use the proper harmonic function for a given dimension to remove local minima.

\section{Panel Method}

In this section, we introduce the panel method, which has been used to solve potential flow of a fluid around bodies of arbitrary shape $[4,7]$. In this method the surface of the body is first 
covered by a finite number of small areas called panels, each of which is dismbuted with source or sink singularities which have uniform density. The dismbuted singularities are used to deflect the oncoming stream so that it will flow around the body. The requirement that the oncoming flow be tangent to every panel at a particular location gives a set of equations which is used to compute the singularity densities on every panel. This panel method has been successfully applied to solve potential flow problems involving two and three dimensional bodies of complex geometry. Note that the path of a point mobile robot matches the path of a single fluid particle through the stream.

\subsection{Single Panel}

In this subsection, we show how a single panel of distributed sources can deflect the oncoming stream. The oncoming stream in Figure 3 is represented by the uniform flow in (12). The single panel in Figure 3 is distributed with uniform sources, with the strength per unit length of $\lambda[4,7]$. The potential at any point $(x, y)$, induced by the sources contained within a small element $d l$ of the panel at $(0, D)$ is

$$
d \phi=\frac{\lambda d l}{2 n} \log r=\frac{\lambda d l}{2 n} \log \left[x^{2}+(y-l)^{2}\right]^{1 / 2} .
$$

The induced potential function by the whole panel is

$$
\phi(x, y)=\frac{\lambda}{4 \pi} \int_{-L}^{L} \log \left[x^{2}+(y-l)^{2}\right] d l .
$$

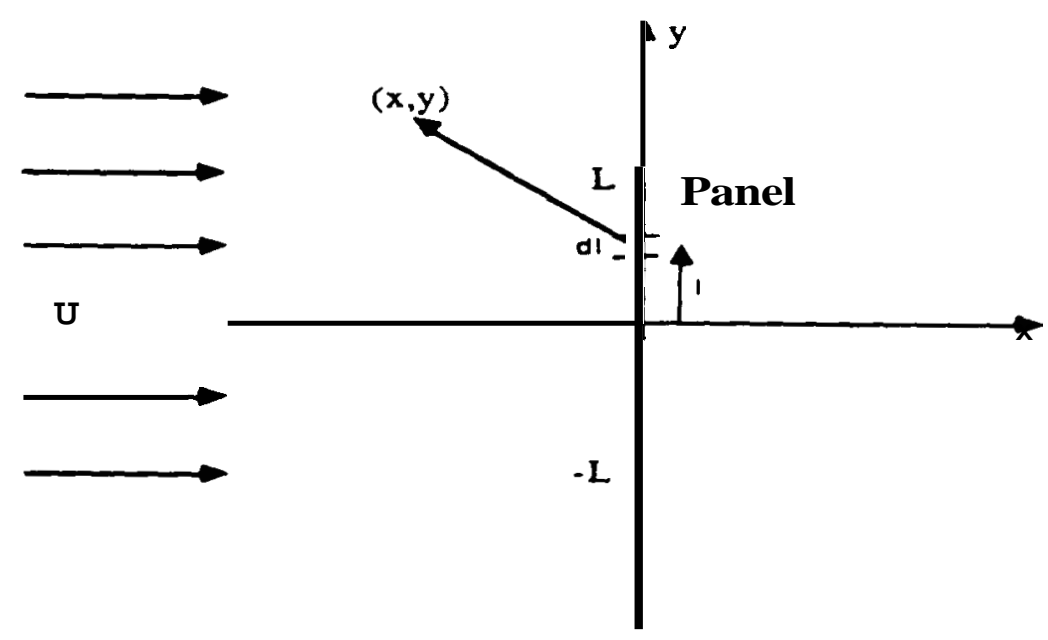

Figure 3: Single Panel

Differentiation with respect to $\boldsymbol{x}$ and y gives, respectively, the expressions for velocity components:

$$
u(x, y)=-\phi_{x}=\frac{\lambda}{2 n}\left(\tan ^{-1} \frac{y+L}{x}-\tan ^{-1} \frac{y-L}{x}\right.
$$




$$
v(x, y)=-\phi_{y}=\frac{\lambda}{2 \pi} \log \frac{x^{2}+(y+L)^{2}}{x^{2}+(y-L)^{2}}
$$

The limiting value of normal velocity $u(x, y)$ in $(17)$ is $u\left(0^{-}, y\right)=-\lambda / 2$ on the left face and $u\left(0^{+}, y\right)=\lambda / 2$ on the right face of the panel when $-L<y<L$. This shows that the source panel of strength $X$ per unit length creates an uniform outward normal velocity of magnitude $\lambda / 2$ at the surface. The tangential velocity $\nu(x, y)$ on the panel starts from zero at the center of panel and increases along the panel surface towards both edges, where the normal velocity is not defined and the tangential velocity becomes infinite. That is, a single panel has a singular point on each edge.

The uniform flow of strength $U$ in Figure 3 flows in the direction of the positive $x$ axis; $a=0$ in (12). The potential and velocity components of this uniform flow are

$$
\begin{aligned}
& \phi(x, y)=-U x \\
& u(x, y)=U, \\
& v(x, y)=0
\end{aligned}
$$

A simple superposition of the two harmonic functions in (16) and (19) results in the uniform flow deflected by the source panel. The total, $x$-direction velocity component of a source panel and a uniform flow at the left face of panel $\left(0^{-}, y\right)$ when $-L<\mathbf{y}<L$, is

$$
u\left(0^{-}, y\right)=U-\lambda / 2
$$

We are interested in this normal velocity on the left face of panel, because the uniform flow is deflected on the left side, but not on the right side. If $U$ equals $\lambda / 2$, then $u\left(0^{-}, y\right)$ becomes zero when $-L<y<L$. That is, the induced normal velocity from a source panel exactly cancels the velocity of oncoming uniform flow on the left face so that the resulting flow becomes tangential to the surface. Both normal and tangential velocities at origin $\left(0^{-}, 0\right)$ are zero. This is a stagnation point, where velocity of a fluid particle becomes zero instantaneously and changes the direction of motion to $-y$ or $y$ direction.

The outward normal velocity of our interest in this example is $-u\left(0^{-}, y\right)$. In hydrodynamics, $u\left(0^{-}, y\right)$ is set to 0 to satisfy the requirement that the oncoming flow must be tangent to a panel. But for our problem of obstacle avoidance, this requirement must be modified as the normal velocity of a panel must be greater than or equal to zero. The requirement for obstacle avoidance can thus be represented as

$$
V_{n}=-u\left(0^{-}, y\right) \geq 0
$$

Three examples of different $V_{n}$ 's are shown in Figure 4. Here $U=1, L=1$ and $\lambda$ is determined by the given $V_{n}$ as in

$$
\lambda=2\left(U+V_{n}\right)
$$


Figures 4-(a), (b), and (c) correspond to $V_{n}=0, V_{,},=1$ and $V_{n}=2$, respectively. The corresponding strengths are $\lambda=2,4$ and 6 from (24). Figures 4-(al), (bl) and (cl) show the profiles of velocity function when $y=0$ and $-5<x<5$; the dotted line represents the $x$ direction velocity induced by source panel along $x$ axis, the dashed line is the velocity of uniform flow, and the solid line corresponds to summation of these two flows. Figures 4-(a2), (b2) and (c2) show trajectories of two fluid particles which start at $(-5.0,-0.1)$ and $(-5.0,0.1)$. Again, note that the motion of a fluid particle can be thought of as the navigation of a point mobile robot which avoids a panel obstacle.

Three important observations can be made from the comparison of these figures. First, the location of the stagnation point moves to the left as $V$,, increases. The stagnation points for these three cases are at $\left(0^{-}, 0\right),(-1,0)$ and $(-1.8,0)$. The stagnation point is where the total velocity (solid line in Figure 4-(al), (bl), and (cl)) becomes zero. Second, the fluid particle moves further away from the panel as $V$,, increases. This provides a tradeoff between economy and safety. As $V_{n}$ becomes larger, a point mobile robot will generally have a longer but a safer path further away from obstacles. Third, the potentials we tested in this section are solutions of Laplace equation for unbounded case. For this unbounded problem, the uniform flow is essential to make a fluid particle move effectively in a desired direction. In this paper, we focus on this unbounded obstacle avoidance problem, even though the potential function can be easily extended to the bounded problem. In the bounded case, the strategy for building an artificial potential is slightly different because the flow of a fluid particle is bounded. We choose to implement the unbounded case only for the sake of convenience.

The two main differences between hydrodynamics and our obstacle avoidance problem are: First, for obstacle avoidance, the normal velocity on the left face of a panel is recommended to be greater than zero to avoid a path which is very close to the obstacle; and second, the obstacle avoidance problem has a final goal point that the robot must reach. We want to move a mobile robot or a manipulator from a starting point to a goal point while avoiding obstacles successfully. Thus, the potential for the obstacle avoidance will be composed of a uniform flow, distributed singularities on panels and a goal singularity (typically sink). In the next section, we discuss the use of multi panels for representing complex obstacles and cluttered environments.

\subsection{Multi Panels}

The use of a set of source/sink panels for representing an arbitrarily shaped obstacle in $\mathbf{2}$ dimensions is illustrated in Figure 5. The obstacle is approximated by a set of panels, which are numbered in the clockwise direction. The details of the panel geometry are shown in Figure 6. Each panel has its own center point with a desired outward normal velocity as an input variable. The boundary points are the intersections of neighboring panels. The angle between panel $i$ and the $x$ axis is 8 , and the angle between the outward normal vector $n_{i}$ of panel $i$ and the $x$ axis is $\beta_{i}$. Then, $\beta_{i}=8,+\pi / 2$.

Let $m$ be the total number of panels. On each of the $\boldsymbol{m}$ panels, whose lengths are usually not equal, sources/sinks of uniform density are distributed. Let $\lambda_{1}, \lambda_{2}, \ldots \lambda_{m}$ represent the source/sink strengths per unit length on these panels. The velocity potential at any point $(x, y)$ in space caused 

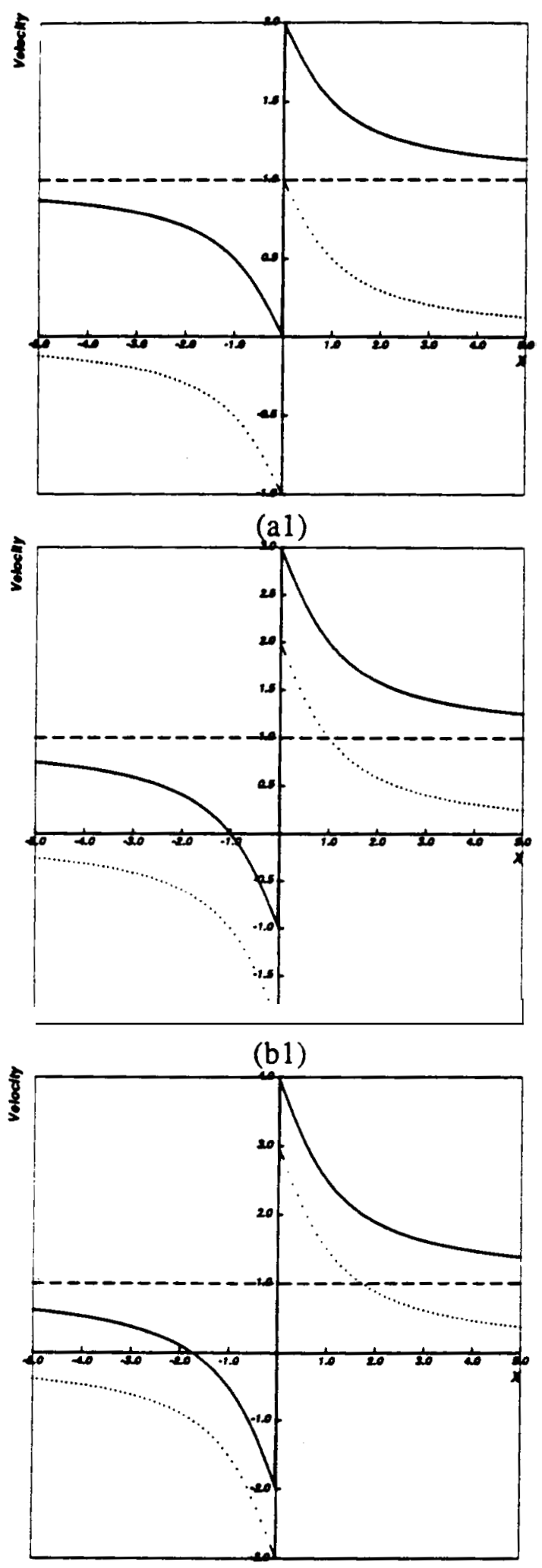

(c1)
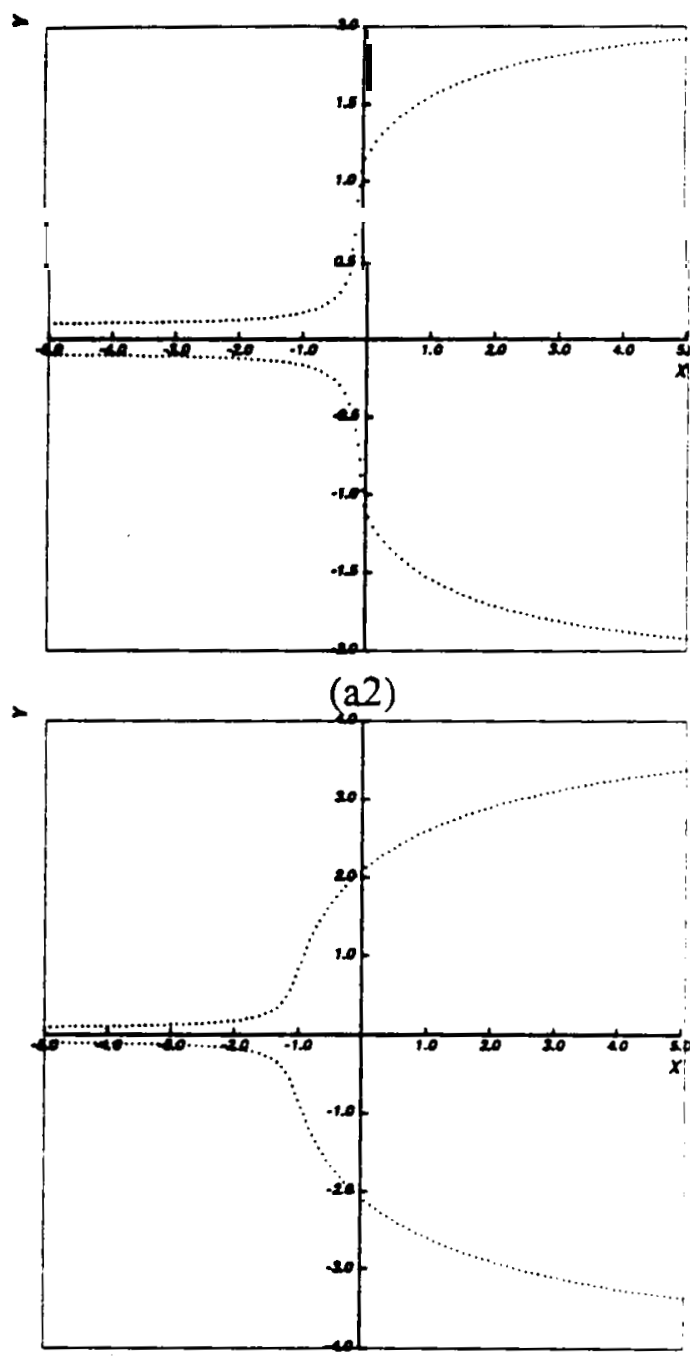

(b2)

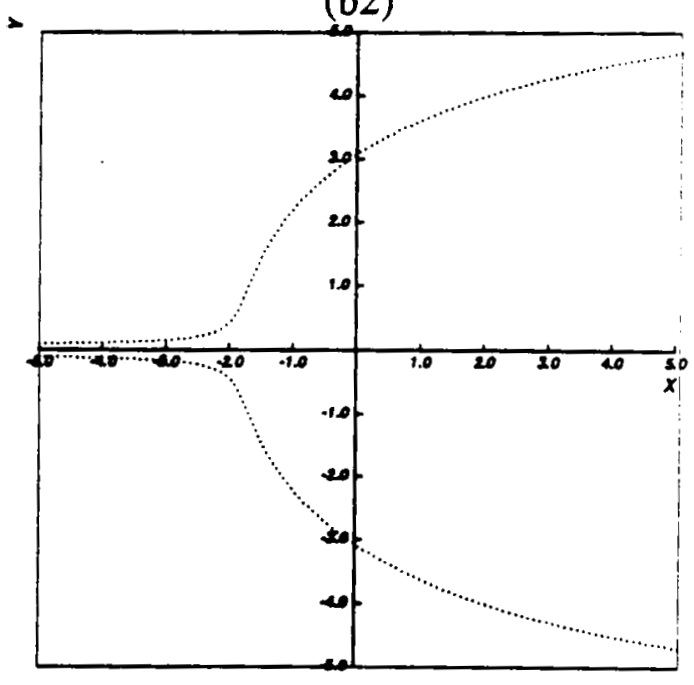

(c2)

Figure 4: Single Panel (a) $V_{n}=0$, (b) $V_{n}=1$ and (c) $V_{n}=\mathbf{2}$; dotted line = velocity by panel source, dashed line $=$ velocity by uniform flow, and solid line $=$ dotted line + dashed line in $(a 1),(b 1)$ and (c1). 


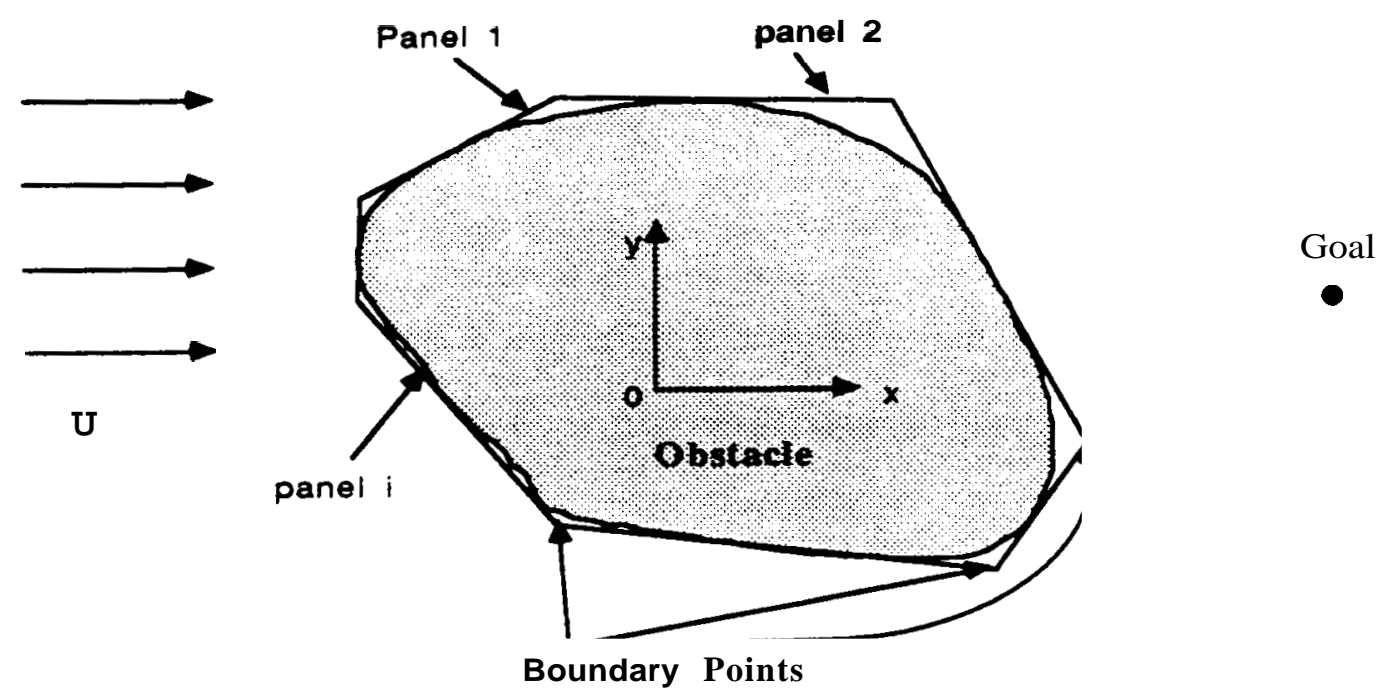

Figure 5: Panel Method

by panel $j$ is

$$
\phi_{j}(x, y)=\frac{\lambda_{j}}{2 \pi} \int_{j} \log R_{j} d l_{j},
$$

where $R_{j}=\sqrt{\left(x-x_{j}\right)^{2}+\left(y-y_{j}\right)^{2}}$

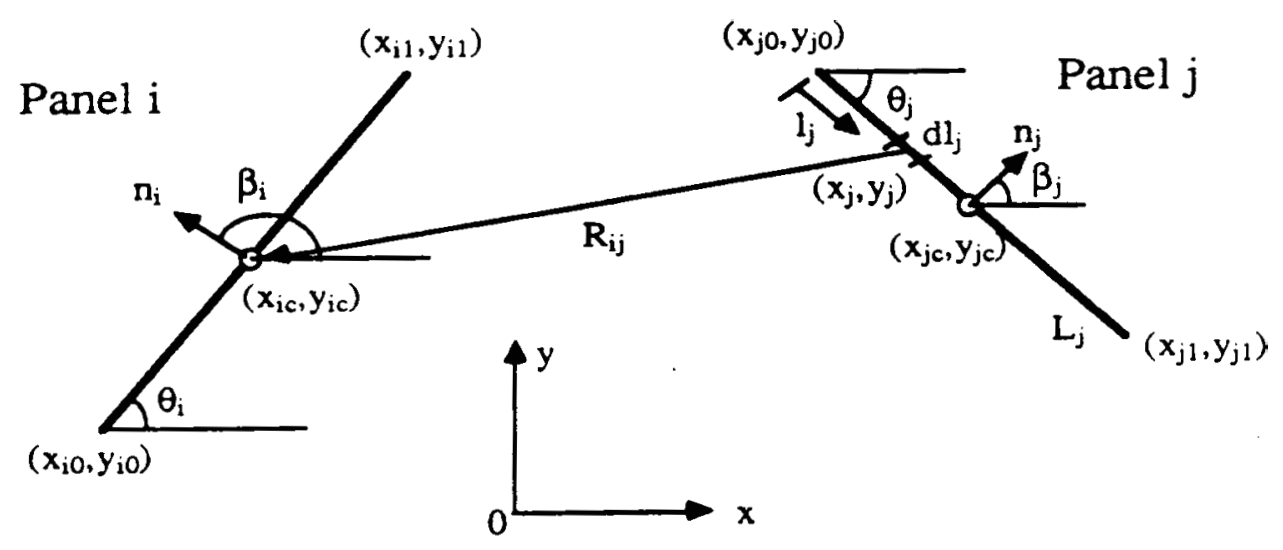

Figure 6: Panel Geometry

The obstacle avoidance problem is to move a robot to a goal while the robot avoids obstacles. We need an attractive potential at this goal, where the potential has only one global minimum. This attractive goal can be represented by a single point singularity of a sink. This sink is like a point drain in a bathtub. Let the goal sink have a strength of $A>0$. Then the potential is

$$
\phi_{g \circ a l}(x, y)=\frac{\lambda_{g}}{2 n} \log R_{g},
$$


where $R,=\sqrt{\left(x-x_{g}\right)^{2}+\left(y-y_{g}\right)^{2}}$ is the distance between the point $(x, y)$ and the goal point $\left(x_{g}, y_{g}\right)$.

The potential of uniform flow in (12), which tends to push the robot to the goal, is rewritten as

$$
\phi_{\text {uniform }}=-U(x \cos \alpha+y \sin \alpha)
$$

where $a$ is the angle between the direction of uniform flow and the $x$ axis.

The total potential due to the obstacles, goal, and uniform flow is

$$
\begin{aligned}
\phi(x, y) & =\phi_{\text {urif orm }}+\phi_{\text {goal }}+\sum_{j=1}^{m} \phi_{j} \\
& =-U(x \cos \alpha+y \sin \alpha)+\frac{\lambda_{g}}{2 \pi} \log R_{g}+\sum_{j=1}^{m} \frac{\lambda_{j}}{2 \pi} \int_{j} \log R_{j} d l_{j},
\end{aligned}
$$

Assuming that the strength of the uniform flow $U$ and the strength of the goal sink $\lambda_{g}$ are given, our objective is to derive the strengths of the $m$ panels. We need at least $m$ independent equations to solve this problem. These $m$ equations are derived from the given outward normal velocities on the $m$ panels. For a single panel, which is normal to uniform flow, and without goal sink, we have derived the relationship between normal velocity on panel and panel strength in (24). A similar, but a little more complicated, expression for the general case can be derived with given desired outward normal velocity on each panel. Let $V_{i}>0$ be the desired outward normal velocity at the center point of panel $i$. Then the resulting $m$ equations are

$$
\frac{\partial}{\partial n_{i}} \phi\left(x_{i c}, y_{i c}\right)=V_{i} \quad i=1,2, \ldots, \text { nn }
$$

These provide $m$ linearly independent equations with variables of $\lambda_{1}, \lambda_{2}, \ldots, \lambda_{m}$. Note that this outward normal velocity is satisfied only at the center point of each panel. The potential at the center point $\left(x_{i c}, y_{i c}\right)$ is

$$
\phi\left(x_{i c}, y_{i c}\right)=-U\left(x_{i c} \cos \alpha+y_{i c} \sin \alpha\right)+\frac{\lambda_{g}}{2 \pi} \log R_{i g}+\sum_{j=1}^{m} \frac{\lambda_{j}}{2 \pi} \int_{j} \log R_{i j} \dot{d} l_{j}
$$

where $R_{i g}$ is the distance between the goal and the center point of panel i, $\left(x_{i c}, y_{i c}\right)$, and $R_{i j}$ is the distance between $\left(x_{i c}, y_{i c}\right)$ and a point on panel $j$ as shown in Figure 6.

By substitution of (30) into (29) and fram the property that the contribution to the normal velocity on panel $i$ by itself is $\lambda_{i} / 2$ (as shown with single panel in previous section), Equation (29) becomes:

$$
\frac{\lambda_{i}}{2}+\sum_{j \neq i}^{m} \frac{\lambda_{j}}{2 \pi} I_{i j}=V_{i}+U \frac{\partial}{\partial n_{i}}\left(x_{i c} \cos \alpha+y_{i c} \sin \alpha\right)-\frac{\lambda_{g}}{2 \pi} \frac{\partial}{\partial n_{i}} \log R_{i g} \quad i=1,2, \ldots, m
$$


where

$$
I_{i j}=\int_{j} \frac{\partial}{\partial n_{i}} \log R_{i j} d l_{j}
$$

By the use of geomemc relations

$$
x_{j}=x_{j 0}+l_{j} \cos \theta_{j} \quad \text { and } \quad y_{j}=y_{j 0}+l_{j} \sin \theta_{j}
$$

$I_{i j}$ can be integrated as [4]:

$$
\begin{array}{rlrl}
I_{i j} & =\frac{1}{2} C \log \left[1+\frac{L_{j}^{2}+2 A L_{j}}{B}-\cos \left(\theta_{i}-\theta_{j}\right)\left[\tan ^{-1} \frac{L_{j}+A}{E}-\tan ^{-1} \frac{A}{E}\right] \text { if } E^{2}>0\right. \\
& =C\left[\log \left|\frac{L_{j}+A}{A}\right|-\frac{L_{j}}{L_{j}+A}\right]+D\left[\frac{1}{A}-\frac{1}{L_{j}+A}\right. & \text { if } E=0
\end{array}
$$

where

$$
\begin{aligned}
& A=-\left(x_{i c}-x_{j 0}\right) \cos \theta_{j}-\left(y_{i c}-y_{j 0}\right) \sin \theta_{j} \\
& B=\left(x_{i c}-x_{j 0}\right)^{2}+\left(y_{i c}-y_{j 0}\right)^{2} \\
& C=\sin \left(\theta_{i}-\theta_{j}\right) \\
& D=-\left(x_{i c}-x_{j 0}\right) \sin 8,+\left(y_{i c}-y_{j 0}\right) \cos \theta_{i} \\
& E=\left(x_{i c}-x_{j 0}\right) \sin \theta_{j}-\left(y_{i c}-y_{j 0}\right) \cos \theta_{j}
\end{aligned}
$$

The parameter $\boldsymbol{E}$ becomes zero when the center point of panel $i$ is on the extension of panel $J$

Now (31) can be written as:

$$
P A=q
$$

where $\Lambda$ is a $m \times 1$ vector of $\lambda_{i}, \mathrm{P}$ is an $m \times m$ mamx and $q$ is an $m x 1$ vector.

We must solve (35) to determine the strengths of the panels. Once these are obtained, we use the following velocity equations to derive a trajectory for real time control.

$$
\begin{aligned}
& u(x, y)=-\phi_{x}=U \cos \alpha-\frac{\lambda_{g}}{2 \pi} \frac{\partial}{\partial x} \log R_{i g}-\sum_{j=1}^{m} \frac{\lambda_{j}}{2 \pi} \int_{j} \frac{\partial}{\partial x} \log R_{i j} d l_{j} \\
& v(x, y)=-\phi_{y}=U \sin \alpha-\frac{\lambda_{g}}{2 \pi} \frac{\partial}{\partial y} \log R_{i g}-\sum_{j=1}^{m} \frac{\lambda_{j}}{2 \pi} \int_{j} \frac{\partial}{\partial y} \log R_{i j} d l_{j}
\end{aligned}
$$

These equations are integrable and the derivation of closed form solutions is omitted for the sake of brevity 


\section{Panel Method for Obstacle Avoidance}

In the previous section, we assumed that the strength of the uniform flow and the strength of the goal sink are given. In this section, we discuss these two potential functions, the stagnation point, and the possible existence of a structural local minimum for a non-point mobile robot and manipulator.

\subsection{Uniform Flow}

The uniform flow is added to derive a more effective potential field fram a starting position of a mobile robot or a manipulator to a goal position. Consider a simple example with a single panel obstacle $(L=1)$ in Figure (3), the goal position at the infinity on the positivex axis and the starting position at $(0,2$.$) . Without an uniform flow, a point mobile robot would move to the positive y$ direction, because the repulsive force of the obstacle is much stronger than the negligible attractive force from the goal. With an uniform flow, the potential around $(0,2$. $)$ will be determined by the strength of the uniform flow plus the strength of a panel source, resulting in a more effective path to the positive $\boldsymbol{x}$ direction.

The direction of the uniform flow $\alpha$ can be determined as follows:

$$
\alpha=\tan ^{-1} \frac{y_{\text {goal }}-y_{\text {start }}}{x_{\text {goal }}-x_{\text {start }}}
$$

Then, the direction of the uniform flow is a straight line connecting the start and goal positions. The relationship between the strength of an uniform flow and the strength of a single source panel is discussed in Section 3.1. Increasing the strength of an uniform flow has the same effect on the resulting trajectory as decreasing the strength of a source panel. Note that the strength of an uniform flow is an input variable but the strengths of panels are determined by (35). If we increase the strength of an uniform flow, the increased strengths of obstacle panels are obtained from (35) to satisfy the given normal velocity $V_{i}$.

\subsection{Goal Sink}

The role of the god sink is to provide a single global minimum over the whole space. In other words, the potential function of (28)has only one global minimum at the location of this goal sink. For a robot to reach this goal, the strength of this goal sink must be large enough. If not, a robot can miss this goal and move to the right in Figure 5, following the uniform flow. To minimize the possibility of collision of the robot with obstacles and the possibility of missing the goal, the strength of the goal sink and the source/sink panels of the obstacle must satisfy the following inequality.

$$
-\lambda_{8}<A<0,
$$


where the obstacle strength $A$ is defined as:

$$
\lambda_{o}=\sum_{i=1}^{m} \lambda_{i} L_{i} .
$$

A positive obstacle strength $(A,>0)$ implies that there is more sink than source. Then the net effect of obstacle panels is attractive and with the resulting potential function, we cannot prevent a robot from colliding with obstacles. If $V_{i}$ in (29) is very small, this positive obstacle strength can be derived. But for an obstacle to provide an repulsive potential, obstacle panels must have more source that sink. On the contrary, if $V_{i}$ is very large, it is possible that $-\lambda_{z}>A$ Then, fluid particles created by obstacles may prevent fluid particles of uniform flow from going into goal sink. This implies that a mobile robot cannot reach the goal and will move to infinity following the uniform flow. To conclude, the above inequality gives the bounds of $V_{i}$, which we input to derive the strengths of panels. The tradeoff is that a large $V_{i}$ implies a safer but less economical (longer trajectory) trajectory away from obstacles.

\subsection{Stagnation Point}

A stagnation point is apoint where the velocity of afluidparticlebecomes zero, but this is an unstable saddle point. We have shown in Section 3.1 that the location of the stagnation point moves away from panel as the strength of source panel increases. Even though a harmonic function does not have local minimum, the harmonic function and the panel method cannot guarantee the obstacle avoidance for a cluttered environment. The panel method we introduced is an approximation, because the given normal velocity is satisfied only on the center point on each panel. This implies that the outward normal velocity may become negative on some points on a panel. We want this negative normal velocity to be caused by only the fluid particles created by panel sources, not by the uniform flow. To satisfy this requirement, the potential must have one stagnation point for every obstacle. Two or more stagnation points imply that there exist fluid particles of uniform flow going into an obstacle. Therefore, the more cluttered environment, the more need there is to ascertain that one obstacle has only one stagnation point. The stagnation points can be found numerically where the following equation is satisfied:

$$
q=\sqrt{u(x, y)^{2}+v(x, y)^{2}}=0
$$

where 4 is called the rate of flow. At Stagnation point, the rate of flow 4 has a local minimum. The stagnation points are typically in the front of each obstacle. This property can reduce the search

space to find stagnation points. If an obstacle has more than two stagnation points, the desired normal velocities on the center points of every panel of that obstacle must be increased until there exists only one stagnation point for each obstacle.

Two alternative solutions to have one stagnation point are to increase the number of panels $m$ and to use a higher order distribution of singularities on each panel. As the number of panels increases, the computation time increases linearly. Infinite number of panels implies a guaranteed solution 
but infinite computation time. In this paper, we used the uniform distribution of a singularity on each panel, which is the simplest form of distributions. The higher order dismbution implies that the outward normal velocity on each panel can be inputed and satisfied at more than two locations on each panel. For instance, the linearly varying distribution has two independent variables which allow to input the desired normal velocities at two locations on each panel. In the following section, we derive control strategy for non-point robots.

\section{Control Strategy for Non-Point Robots}

For a point mobile robot, which could be thought of as a fluid particle, there is no need to calculate the distance from obstacles, since we have a closed form potential function which can generate a trajectory successfully. But this potential cannot guarantee a collision free trajectory for a non-point mobile robot or a manipulator without an elegant control strategy. These robots are represented as a straight line or a set of straight lines. The end-effector of a manipulator or the leading point of a mobile robot is called the primary control point in Figure 7 . This primary control point is always under the control of artificial potential and can be considered as a point mobile robot. For the other parts of a robot, we need to calculate the distance from obstacles. The distance can be easily obtained without increasing computational time much, since all obstacles are approximated by polygons (sets of panels) [21].

If the part of a robot is within a minimum allowable distance $\delta$ from obstacles, the location of the closest point on that part of a robot become a secondary control point, and the corresponding point on a panel of obstacles becomes an obstacle point. This minimum distance 6 is an input variable. A robot can have more than one secondary control point when it is between more than two obstacles as in Figure 7 or when it is interacting with a concave obstacle.

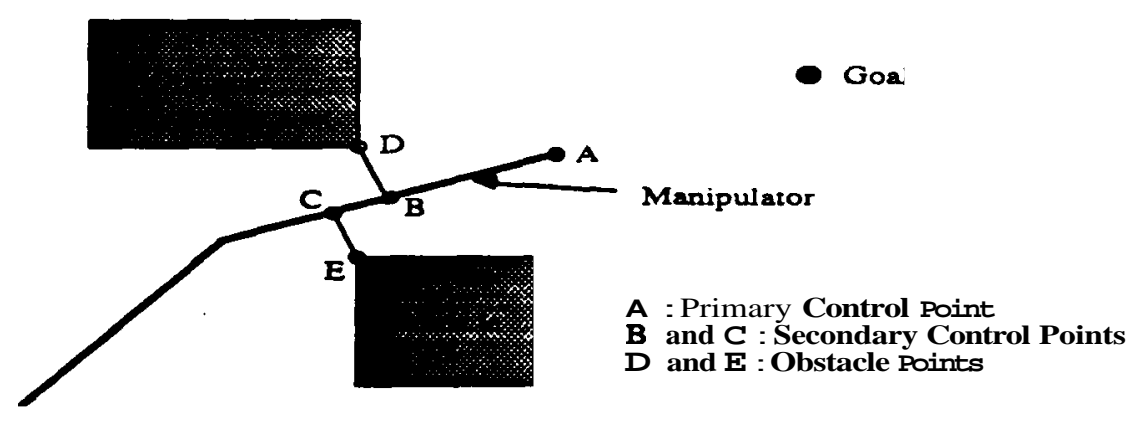

Figure 7: Control Point and Obstacle point

Depending on the number of secondary control points, arr strategy for real time control of a robot is divided into two different operations.

\section{(1) Potential Operation:}

When there is no secondary control point, the primary control point is controlled by the artificial potential derived in Section 3; while the other parts of a robot are controlled by the same potential or are used to optimize some performance criteria in [23]. 


\section{(2) Obstacle Operation:}

When the number of secondary control points is nonzero, then a new harmonic function with point sources at obstacle points is used to repel secondary control points while the primary control point is controlled by the same potential function as used in the potential operation. That is, we have two different potential fields. The second potential function $\phi_{0}$ to control the secondary control points is

$$
\phi_{o}(x, y)=\sum_{i=1}^{n_{c}} \frac{\lambda_{c i}}{2 \pi} \log R_{c i}
$$

where $n_{c}$ is number of the secondary control points, $\lambda_{c i}$ is the strength of the point source at obstacle point $i$, and $R_{c i}$ is the distance from obstacle point $i$ to $(x, y)$. Note that the secondary control point is the closest point from the obstacle within the minimum allowable distance 6 . The secondary control point is updated every period until it goes out of the minimum allowable distance from obstacle.

In the next two subsections, we show simulation results with a bar-shaped mobile robot and a 3 dof redundant planar manipulator, respectively.

\subsection{Mobile Robot}

A bar-shaped mobile robot has three degrees of freedom $(x, y, \eta)$ in a plane, where $(x, y)$ is the location of the leading point (primary control point) on the bar and $\eta$ is the angle between the bar and the $\boldsymbol{x}$ axis. We want this robot to move from $\left(x_{0}, y_{0}\right)$ to $\left(x_{g}, y_{g}\right)$. This robot has one redundant dof for the given task and this redundancy is used to avoid obstacles.

During the potential operation, the displacement of the primary control point and the angle per period can be obtained as

$$
\Delta x=u(x, y), \quad \Delta y=v(x, y) \quad \text { and } \quad \Delta \eta=\tan ^{-1}[v(x, y) / u(x, y)]-\eta,
$$

where $u(x, y)$ and $v(x, \mathrm{y})$ are derived in (36) and (37). As a fluid particle gets closer to the obstacle, a larger velocity is induced. This is because a fluid particle close to an obstacle has a longer trajectory to travel in the same time than particles away from an obstacle. Therefore, we cannot use the value obtained from (43). The displacements are adjusted by the directional information and the maximum possible displacement $d_{\max }$ per period according to:

$$
\begin{aligned}
& \Delta x=d_{\max } \cos (\eta+\Delta \eta) \\
& \boldsymbol{A y}=d_{\max } \sin (\eta+\Delta \eta) .
\end{aligned}
$$

where $d_{\max }$ is the maximum possible displacement per period. This normalizanon does not change $\eta$. Assuming that the angle change has a maximum saturation value per period, we need to readjust the normalized displacement if $\Delta \eta$ is larger than $\Delta \eta_{\max }$;

$$
H=\Delta \eta_{\max } /|\Delta \eta|
$$




$$
\begin{aligned}
& \Delta x:=\Delta x * H \\
& \boldsymbol{A y}:=\boldsymbol{A y} * H \\
& \Delta \eta:=\Delta \eta * H
\end{aligned}
$$

For the obstacle operation, the second potential $\phi_{o}$ to control secondary control points is obtained from (42). The displacement of primary control point is determined the same way as in (44) and (45), but the angle $\eta$ is determined by the new potential $\phi_{o}$. Assuming the primary control point is fixed, we can obtain $\Delta \eta$ by the potential on the secondary control points.

The obstacle and the panel numbering (clockwise and $m=16$ ) for an example problem are shown in Figure 8, and the simulation result is shown in Figure 9. The initial position of the primary point is at $\left(x_{0}=1.2, y_{0}=8.5, \eta_{0}=0.0\right)$ and final goal is at $\left(x_{g}=8.0, y_{8}=\mathbf{2 . 0}\right)$. Note that the unit of distance is meter and the unit of angle is radian. Other input variables are

$$
U=1.0, \quad \lambda_{g}=30 \quad \text { and } \quad V_{i}=0.5 \quad i=1,2, \ldots, 16 \text {. }
$$

The strengths of panels obtained from (35) are shown in Table 1. Table 1 shows that panels which confront the uniform flow become sources to deflect the uniform flow and other panels become sinks to absorb flows created by source panels, but they satisfy the given normal velocity at the center points of each panel. Note that the derived obstacle strength $\lambda_{0}$ satisfies the inequality condition in (39).

With a concave obstacle, the bar-shaped mobile robot successfully navigated to the goal as shown in Figure 9. This result is not a surprise since we used the harmonic potential. The resulting potential has one stagnation point around $(2.5,6.5)$. If the robot gets to this stagnation point, then it will make a sharp left or right turn. We have to make the robot choose one direction. For most obstacles, the stagnation point implies that either direction is fine because both trajectories have almost the same length. With the minimum allowable distance 6 from obstacle surface equal to 0.2, this example shows only the potential operation. Obstacle operation will be shown in the next example with a redundant manipulator.

\begin{tabular}{||c|r||c|r||c|r||c|r||}
\hline panel & strength & panel & strength & panel & strength & panel & strength \\
\hline 1 & -4.955 & 5 & -1.269 & 9 & 3.931 & 13 & 4.632 \\
\hline 2 & -8.487 & 6 & -5.408 & 10 & 2.626 & 14 & 5.390 \\
\hline 3 & -8.572 & 7 & 5.457 & 11 & 2.489 & 15 & -5.449 \\
\hline 4 & -5.077 & 8 & 4.719 & 12 & 3.832 & 16 & -1.364 \\
\hline
\end{tabular}

Table 1: Panel Strengths of Obstacle-1 (obstacle strength $=-7.503$ )

\subsection{Redundant Manipulator}

A 3 dof redundant manipulator is used as another example of obstacle avoidance in an environment of multiple obstacles in Figure 10. The total number of panels is $\boldsymbol{m}=\mathbf{2 4}$ (each obstacle has 12 


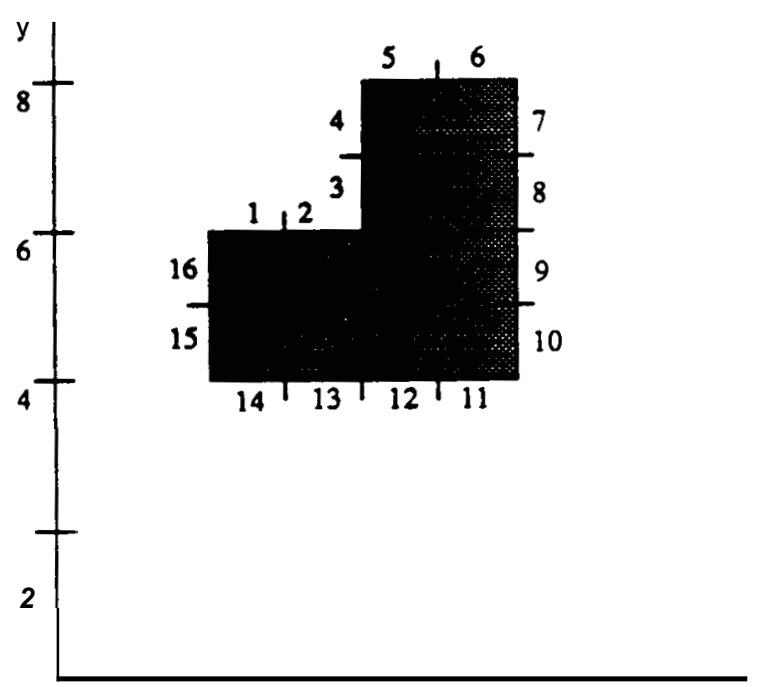

Figure 8: Obstacle-1 $(m=16)$

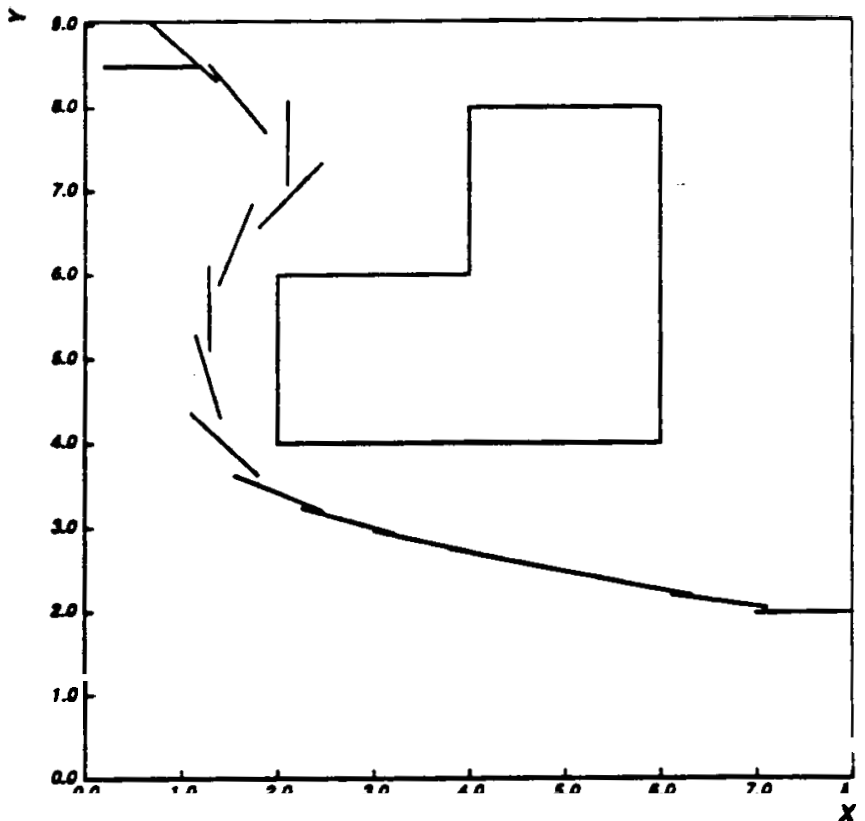

Figure 9: A Bar-shaped Mobile Robot (The average CPU ame per period on VAX 780 is about 10 msec.) 
panels). The task is to move end-effector from $(-2.0,8.0)$ to the god at $(5.0,9.0)$. The base of manipulator is at $(\mathbf{- 2 . 0 , 2 . 0})$. The initial angle between the third link and the $\boldsymbol{x}$ axis is 10 degrees. To perform this task, the end-effectorhas to pass through the aperture between the two rectangular obstacles. The input variables are given as:

$$
U=1.0, \quad \lambda_{g}=30 \quad \text { and } \quad V_{i}=1.0 \quad i=1,2, \ldots, 24
$$

Table 2 shows the strengths of panels obtained from (35). Here again, we observe that panels that confront the uniform flow become sources and others become sinks. The obstacle strengths $\lambda_{0}$ of both obstacles and their summation satisfy the inequality condition in (39).

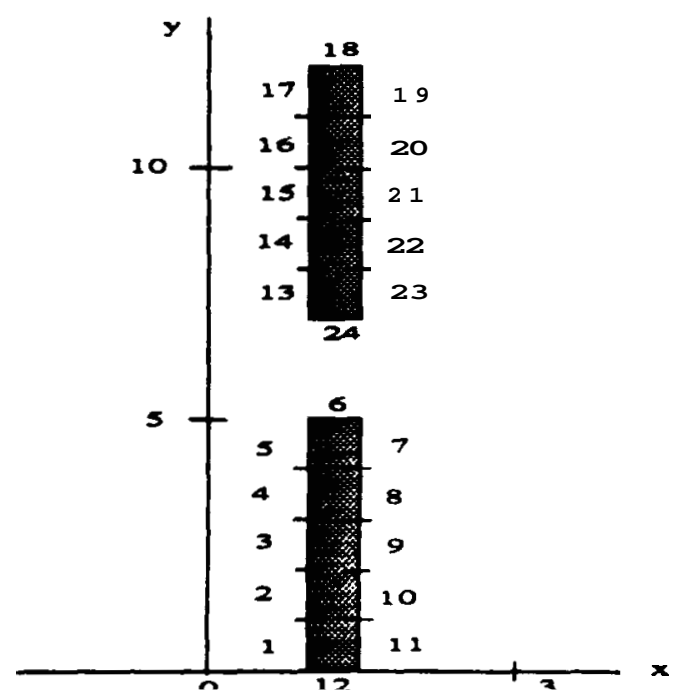

Figure 10: Obstacle-2 ( $m=\mathbf{2 4})$

For the potential and the obstacle operation, the displacement of the end-effector (the primary control point) is determined by

$$
\Delta x_{e}=u(x, y) \quad \text { and } \quad \Delta y_{e}=v(x, y) .
$$

After normalization of the above displacements, the displacement of the joint angles (net motion) to satisfy this end-effector displacement is determined by

$$
\Delta \theta_{p}=J_{e}^{+} \Delta X_{e}
$$

where $J_{e}^{+}$is a Moore-Penrose pseudoinverse of end-effector Jacobian $J_{e}$ and $\Delta X_{\epsilon}=\left[A x, . \Delta y_{e}\right]^{T}$.

During the potential operation, due to the self motion or null motion of a redundant manipulator, there are an infinite number of ways for the end-effector to follow the gradient of the potential field obtained. This self motion can be controlled by some optimizing function. For instance, it is possible to maximize the manipulability [23] while the end-effectorfollows the gradient of the 


\begin{tabular}{||c|r||c|r||c|c||c|r||}
\hline panel & strength & panel & strength & panel & strength & panel & strength \\
\hline 1 & -14.014 & 7 & 14.849 & 13 & -22.325 & 19 & 17.999 \\
\hline 2 & -18.239 & 8 & 17.990 & 14 & -28.297 & 20 & 24.802 \\
\hline 3 & -20.175 & 9 & 18.054 & 15 & -29.466 & 21 & 27.55 \\
\hline 4 & -20.123 & 10 & 16.096 & 16 & -26.807 & 22 & 26.386 \\
\hline 5 & -17.267 & 11 & 11.748 & 17 & -20.224 & 23 & 20.479 \\
\hline 6 & 0.780 & 12 & -0.700 & 18 & -0.532 & 24 & -2.342 \\
\hline
\end{tabular}

Table 2: Panel Strengths of Obstacle-2 (lower obstacle strength $=-11.040$ and upper obstacle strength $=-11.332$ )

artificial potential. In this example, the self motion is controlled by the same potential field where motion of the end point of each link is determined by the potential on that point.

For the obstacle operation, an additional potential is obtained from (42). The null motion which moves the secondary control points away from the obstacle points is calculated based on the algorithm proposed in [15]. The joint displacement for this null motion is

$$
\Delta \theta_{h}=\sum_{i=1}^{n_{c}}\left[J_{c i}\left(I-J_{e}^{+} J_{e}\right)\right]^{+}\left(\Delta X_{c i}-J_{c i} J_{e}^{+} \Delta X_{e}\right),
$$

where $\Delta X_{c i}$ is the desired displacement at the ith secondary control point, $I$ is a $3 \times 3$ identity mamx, and $J_{c i}$ is a $2 \times 3$ secondary control point Jacobian mamx. Note that the above $\Delta \theta_{h}$ is in the null space of the Jacobian $J_{e}$.

Now we have the total motion $\Delta \theta$ which is a sum of the net motion to reach the goal and the null motion for obstacle avoidance.

$$
\Delta \theta=\Delta \theta_{p}+\Delta \theta_{h}
$$

Assuming the total energy consumption per period of the manipulator is fixed, we adjust the net and the null motions in (55) as follows:

$$
\begin{aligned}
H & =\Delta \theta_{\max } /\|\Delta \theta\| \\
\Delta \theta_{p} & :=\Delta \theta_{p} * H \\
\Delta \theta_{h} & :=\Delta \theta_{h} * H
\end{aligned}
$$

In Figure 11, the end-effector motion is very small in the middle of trajectory after passing the aperture. This is because there is a large null motion to avoid the upper obstacle in the obstacle operation. The minimum allowable distance 6 from obstacle was set to 0.2 . The simulation shows that the end-effector of the manipulator reaches the goal successfully while the links avoid the obstacles.

In the following section, we discuss the structural local minimum which appears for non-point robots. 


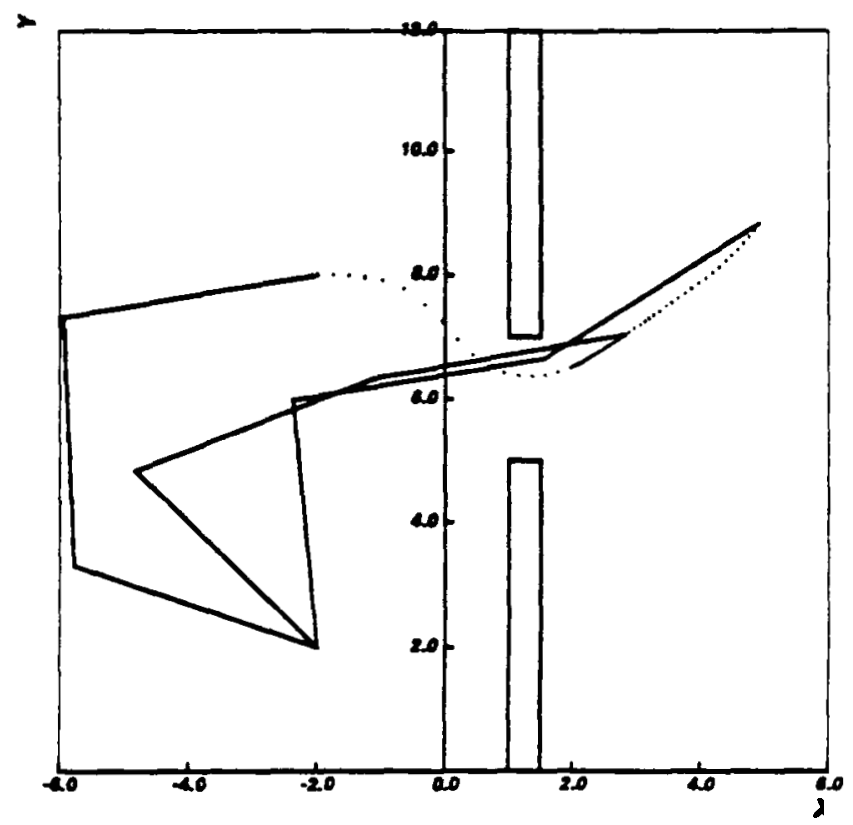

Figure 11: A Redundant Manipulator (The average CPU time per period on VAX 780 is about 26 msec.)

\section{Structural Local Minimum}

An artificial potential of harmonic functions does not have a local minimum other than one global minimum at the goal position. This implies that for a point mobile robot, there exists only one global minimum at the goal. However, this does not imply that a mobile robot and a manipulator, which cannot be approximated as a point, can move to the goal without failure. This is because the artificial potential approach for rel time control is local and not global. The structural local minimum for a non-point robot can be defined as:

The structural local minimum is a static equilibrium where the robot cannot move any more with a current artificial potential.

Two examples of this structural local minimum are shown in Figure 12, where the goal sink attracts a robot while the obstacle panels repel a robot. This is not a deficiency of a robot. For instance, even a rope manipulator, which has infinite dof, cannot reach the goal if it chooses a wrong trajectory derived by artificialpotential. Increasing the degrees of freedom of a manipulator helps but cannot be an ultimate solution [21]. If the goal is located out of reachable space, it cannot be reached by any trajectary. This is not a structural local minimum. Only when there exists at least one possible trajectory and the robot is in static equilibrium with repulsive forces from obstacles and attractive force from the goal sink, do we say that the robot is in a structural local minimum.

This structural local minimum results from all local approaches. Therefore, one solution is to 


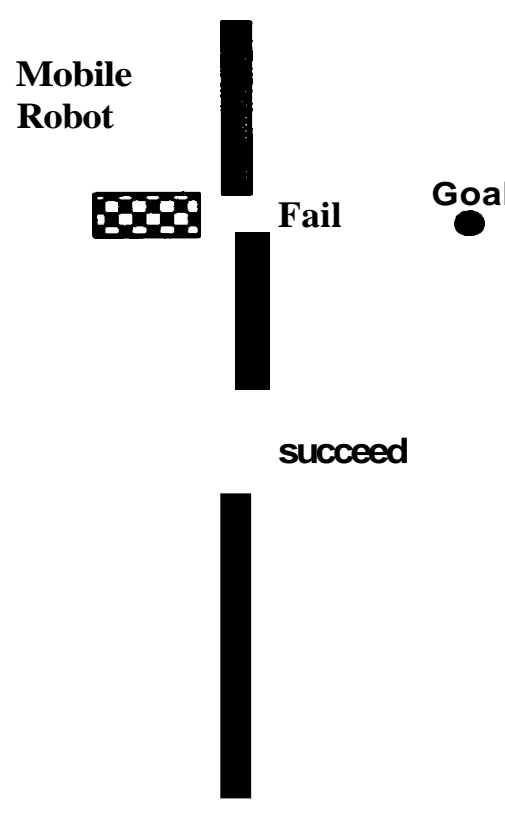

(A)

\section{DOF Planar Manipulator}

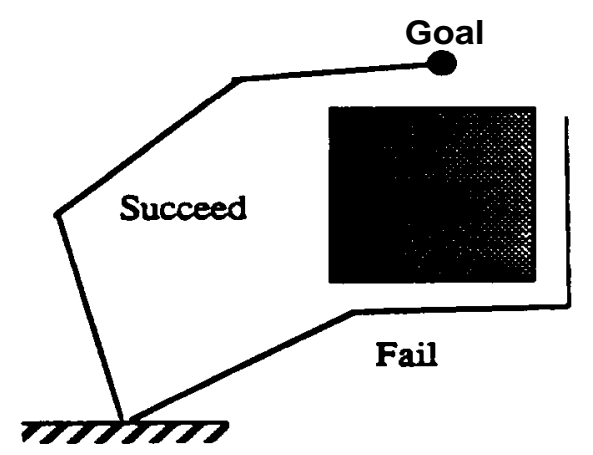

make it global by using artificial potential in configuration space as in [17, 19, 22]. The other solution is based on a mal and error approach and involves incorporating an artificial obstacle [13]. The artificial obstacle is used to remove one possible trajectory that causes structural local minimum. With this method, several paths may be tested until a robot reaches the goal.

\section{Conclusion}

In this paper, we introduced the harmonic potential function for building potential fields for obstacle avoidance. We proposed the use of harmonic functions to eliminate local minima even for a cluttered environment and for concave obstacles. We also introduced the panel method for modeling obstacles. A point mobile robot is treated like a fluid particle in a potential flow. For a point mobile robot, if we use the panel method, we don't need to calculate the distance from obstacles. The panel method provides a closed form potential function and can be easily implemented. Its computation time increases linearly as the number of obstacles and the number of dof of a robot increase. The shape of a robot can be easily considered during real-time control.

For non-point robots, we developed control strategy under the potential field obtained by panel method and harmonic functions and it was tested with a mobile robot and a redundant manipulator for a $\mathbf{2}$ dimensional problem. Application of our approach to $\mathbf{3}$ dimensions is also possible with 3 dimensional harmonic functions. Furthermore, our potential based approach can be applied to the global path planning problem in task space as well as configuration space. We showed 
with an example that trajectory of a point mobile robot changes as the strength of panel changes. The problem of finding the best trajectory which optimizes some criterion (say, energy) can be formulated as a problem of finding the best strengths of panels which optimizes the same criterion.

\section{References}

[1] S. Akishita, S. Kawamura and K. Hayashi, "Laplace Potential for Moving Obstacle Avoidance and Approach of a Mobile Robot," I990 Japan-USA Symposium on Flexible Automation, A Pacific Rim Conference,pp. 139-142, Kyoto, Japan, 1990.

[2] J. Bartaquand, B. Langlois, J.-C. Latombe, "Robot Motion Planning With Many Degrees of Freedom and Dynamic Constraints," Robotics Research, the 5th international symposium, 1989.

[3] R.A. Brooks, Solving the Find-Path Problem by Representing Free Space as Generalized Cones, MIT AI Memo no. 674, May, 1982.

[4] C.-Y. Chow, An Introduction to ComputationalFluid Mechanics, John Wiley \& Sons, 1979.

[5] R. Colbaugh, H. Seraji and K.I. Glass, "Obstacle Avoidance for Redundant Robots Using Configuration Control," J. of Robotic Systems: vol. 6, no. 6, pp. 721-744, 1989.

[6] C.I.Connolly, J.B. Burns and R. Weiss, "Path Planning Using Laplace's Equation," IEEE conf. on Robotics and Automation, PP. 2102-2106.1990.

[7] A.M. Kuethe and C.-Y. Chow, Foundations ofAerodynamics: Bases ofAerodynamic Design, John Wiley \& Sons, thed., 1986.

[8] C.R. Chester, Techniques in Partial DifferentialEquation, McGraw-Hill, 1971.

[9] Z.YGuoand T.C. Hsia, "JointTrajectory Generation for Redundant Robots in an Environment with Obstacles," IEEE conf. on Robotics and Automation, pp. 157-162, 1990.

[10] N. Hogan, "Impedance Control: an approach to manipulation," ASME Trans. of Dynamic Systems, Measurement, and Control, vol. 107,pp. 1-24,March, 1985.

[11] O .Khatib. "Real-Time Obstacle Avoidance for Manipulators and Mobile Robots," Int. J. of Robotics Research, vol. 5, no. 1, pp. 90-98, 1986.

[12] P. Khosla and R. Volpe. "Superquadric Artificial Potentials for Obstacle Avoidance and Approach," IEEE conf. on Robotics and Automation, pp. 1778-1784, 1988.

[13] J.-0. Kim and P. Khosla, "Real-Time Obstacle Using Harmonic Potential Functions," IEEE conf. on Robotics and Automation, pp. 790-796,Sacramento, CA, 1991.

[14] T. Lozano-Pérez, "Spatial Planning: A Configuration Space Approach," IEEE transactions on Computers, vol. C-32(2), 1983. 
[15] A.A. Maciejewsti and C.A. Klein, "Obstacle Avoidance for Kinematically Redundant Manipulators in Dynamically Varying Environments," The International Journal of Robotics Research, vol. 4, no. 3, pp. 109-117, Fall, 1985.

[16] L.M. Mille-Thomson, TheoreticalHydrodynamics. New York: The Macmillan Company, 5th ed., 1968.

[17] M. Okutomi and M. Mori, "Decision of Robot Movement by Means of a Potential Field," Advanced Robotics, vol. 1, no. 2, pp. 131-141,1986.

[18] C.R. Rao and S.K.Mitra, Generalized Inverse of Matrices and its Application. John Wiley and Sons, 1971.

[19] E. Rimon and D.E.Koditschek, "Exact Robot Navigation in Geomemcally Complicated but Topologically Simple Spaces," IEEE int. conf on Robotics and Automation, pp. 1937-1942, 1990.

[20] J.M. Robertson, Hydrodynamics in Theory and Application. Prentice-Hall Inc., 1965.

[21] R. Volpe and P. Khosla, "Manipulator Control with Superquadric Artificial Potential Functions: Theory and Experiments" IEEE Transactions on Systems, Man, and Cybernetics; Special Issue on Unmanned Vehiclesand Intelligent Systems, November 1990.

[22] C.W. Warren, "Global Path Planning Using Artificial Potential Fields," IEEE inr. conf. on Robotics and Automation, pp. 316-321, 1989.

[23] T. Yoshikawa, "Analysis and Control of Robot Manipulators with Redundancy," Robotics Research: The first int. symp., MIT press, pp. 735-747, 1984. 
